\title{
Childhood Obesity: A Comprehensive Review of Epidemiology, Aetiopathogenesis and Management of This Global Threat of the $21^{\text {st }}$ Century
}

\author{
Kulvinder Kochar Kaur ${ }^{1 *}$, Gautam Allahbadia ${ }^{2}$ and Mandeep Singh ${ }^{3}$ \\ ${ }^{1}$ Scientific Director, Dr Kulvinder Kaur Centre for Human Reproduction, Jalandhar, Punjab, India \\ ${ }^{2}$ Scientific Director, Rotunda-A Centre for Human Reproduction, Mumbai, India \\ ${ }^{3}$ Consultant Neurologist, Swami Satyanand Hospital, Jalandhar, Punjab, India
}

*Corresponding Author: Kulvinder Kochar Kaur, Scientific Director, Dr Kulvinder Kaur Centre for Human Reproduction, Jalandhar, Punjab, India.

Received: August 17, 2019; Published: August 27, 2019

\begin{abstract}
There is increasing prevalence of obesity in childhood and adolescence with WHO paying marked attention for the global prevention of same with the public health problem it has assumed. Hence we decided to do a comprehensive review on this topic. We searched pubmed articles with the MeSH terms childhood obesity, adolescent obesity, causes of childhood obesity, management and epidemiology criteria of defining the same in various geographica areas of the world. In this aspect we found 10,300 articles from 1960-2018 but of these only 90 articles were there after ruling out duplicate ones which we decided to use for the review. No metaanalysis was done. We selected articles pertaining to different geographical areas representing diagnostic criteria from countries like China, a large developing country to countries like Japan, USA, UK which are more developed. BMI and WC percentile curves in China, POW and BMI reference range for Japan, International Obesity Task Force (IOTF ) criteria were used in Chilea, Singapore, Holland, USAand Great Britain besides using WHO updated BMI criteria. Success of implementing various educational, medical and public health interventions in some developed countries has resulted in plateauing of this increasing incidence yet in developing countries like china it is still a challenge. Further role of increasing prevalence of childhood obesity related to the developmental hypothesis of health and disease [DOHa D), maternal resource hypothesis, with effect of intrauterine fetal environment affecting fetal metabolism, infant feeding all influence development of childhood obesity and importance of tracking obesity from infancy to childhood and from childhood to adulthood for prevention of development of severe type2diabetesand other metabolic syndrome related diseases. Hence prevention was the most important factor for controlling this epidemic, besides knowledge regarding family environment and obesity of parents has a direct influence on children weight gain, role of increased television watching and munching simultaneously has to be kept in mind to have better preventative measures besides finding cause of loss of inhibitory control. Once therapy is considered lifestyle measures, diet therapy, avoidance of pharmacotherapy are the main principles. Still need of bariatric surgery is there in limited morbidly obese children and adolescents.
\end{abstract}

Keywords: Childhood obesity; DOHaD; WHO;IOTF; BMI; Maternal Resource Hypothesis.

\section{Introduction}

In our previous articles we have emphasized on the aetiopathogenesis along with medical treatment, updates on medical treat- ment and bariatric surgery [1-3]. Although we did touch on childhood obesity but considering the markedly increasing incidence of childhood and adolescent obesity we have tried to consider the

Citation: Kulvinder Kochar Kaur., et al. "Childhood Obesity: A Comprehensive Review of Epidemiology, Aetiopathogenesis and Management of This Global Threat of the $21^{\text {st }}$ Century". Acta Scientific Paediatrics 2.9 (2019): 56-66. 
epidemiology, prevention of childhood and adolescent obesity from different geographical regions of the world along with understand the aetilology of this to help preventing the development of lifestyle diseases like type2 diabetes mellitus (T2DM), hypertension, hyperlipidemia related to obesity besides the development of various cancers.

\section{Role of Maternal Resource Hypothesis}

Over the past century there has been a socio environmental evolution like reduced pathogenic load, reduced physical activity along with improved nutrition, which led to cumulative increments in maternal energy resources i. e body mass and adiposity associated with reduction in energy expenditure and metabolic control. Because of these there was decreased competition between maternal and fetal energy demands and increased the avail ability of energy substrates to the intrauterine milieu. This change in maternal-conceptus energy partitioning stimulated fetal $\beta$-cell and adipocyte hyperplasia and hence led to endurance of competitive dominance of adipocytes over other tissues in the acquisition along with sequestering of nutrient energy through intensified insulin secretion along with hyperplastic adiposity. At menarche, the competitive dominance of adipocytes was further amplified through hormone induced adipocyte hyperplasia and weight induced reduction in physical activity. These metabolic and behavioral effects got propagated progressively once obese, inactive, metabolically compromised women produced progressively larger, more inactive metabolically compromised children. Thus the evolution of human energy metabolism was significantly changed. The phenotypic evolution got exaggerated by increased incidence of caesarean sections, that allowed both larger fetuses as well as metabolically compromised mothers who produced them to survive and reproduce. This led to natural selection getting iatrogenically replaced by artificial selection, and the frequency of obese, inactive metabolically compromised phenotypes increased in the global population. Thus by the $20^{\text {th }}$ century a metabolic tipping point got reached at which the postprandial insulin response was so intense and the relative number of adipocytes so large, and inactivity so pervasive that the competitive dominance of adipocytes in the sequestering of nutrient energy was inevitable and obesity was unavoidable [4].

\section{Epidemiology}

Childhood obesity, as defined by an excessive fat accumulation status in children [5], has become one of the most serious public health challenges in the $21^{\text {st }}$ century, allover the world [6]. Appro- ximately 170 million children ( $<18 y$ old) suffer from the physical and physiological consequences caused by overweight or obesity, resulting in reduced life quality along with greater risk of developing noncommunicable diseases like cardiovascular diseases, type2diabetes and cancers in their later adulthood. Though still there are regions which are still struggling with child hunger like in Southeastern Asia and Sub Saharan Africa, childhood obesity has already reached endemic proportions by spreading to developing countries like China [7].

\section{In China}

At the population level, percentile curve is a useful tool for monitoring and screening obesity among school aged children and adolescents. The most commonly used indicator is body mass index (BMI). Overweight and obesity in children are generally defined respectively based on the $85^{\text {th }}$ percentile and $95^{\text {th }}$ percentile of BMI among children. Though BMI has been used to show the weight for height relationship and can provide to some extent an estimate of body fat percentage, BMI by itself cannot reflect the fat distribution very comprehensively, like abdominal fat depots [8].

Visceral fat deposition signifies abdominal obesity, and is associated with greater risk of metabolic complications like hyperlipidemia and diabetes [9]. The indicators used for measuring abdominal obesity are waist circumference (WC) and related indices like waist to height and waist to hip ratio [10]. It is easier to measure WC that is simple and convenient for measuring accumulation of abdominal fat [. Since children are growing with bodies developing over years it may not be appropriate. Earlier it has been suggested that age and gender specific $W C>=90^{\text {th }}$ percentile be used as the threshhold for abdominal obesity. Different childhood BMI and WC reference percentiles have been made in China at local and national levels based on clinical and population practice [11]. Of these the most nationally representative BMI and WC percentiles were based on a large cross-sectional school based investigation conducted in 2005 called the Chinese National Survey on Students Constitution and Health (CNSSCH) [11]. In this participants were students from primary and high schools who were between ages 7-18yrs. Main limitation was that percentiles were based only on cross-sectional data and thus this may limit the reflection of the real BMI and WC growth patterns with age $[35,38]$. Also the childhood growth patterns changes with time, economic and social development, thus there is need for regular updating, and the CNSSCH BMI and WC re- 
ference percentiles used presently were based on data in the year 2005 and hence its usage might not be practical.

There was another prospective household based study namely the China Health and nutrition Survey (CHNS), that has been conducted in years 1989, 1997, 2000, 2004, 2006, 2009and 2011 and it covered nine provinces which had different geographies, economic development level along with health indicators. This study had both the merits of being nationally representative and longitudinal follow upquality, giving better reflection of growth patterns.

Thus to construct the percentile reference values of BMI and WC and then calculate the prevalence of overall and abdominal obesity for Chinese children and adolescents, Song., et al. 2016 studied a total of 5062anthropometric records for children and adolescents aged from 7-18yrs (2679 boys and 2383 girls). These participants were recruited as part of the national representative CHNS. Age, gender, weight, height and WC were assessed. Smoothened BMI and WC percentile curves and values for the $3^{\text {rd }}, 5^{\text {th }}, 10^{\text {th }}, 25^{\text {th }}, 50^{\text {th }}$, $75^{\text {th }}, 85^{\text {th }}, 90^{\text {th }}, 95^{\text {th }}$ and $97^{\text {th }}$ percentiles were constructed by using the Lambda -mu-sigma (LMS) method. The prevalence estimates of the overall and abdominal obesity were calculated by using the cutoffs from CHNS study along with the CNSSCH study conducted in 2005 which was based on WHO and US Centre for Control and prevention, respectively. Difference between prevalence estimates was tested using a McNemar's test and the agreement between these prevalence estimates was calculated by using the Cohen's kappa coefficient. They found that the prevalence values of overall obesity based on the cutoffs from CHNS and CNSSCH were an almost perfect agreement levels in boys ( $\kappa=0.93)$. However, in girls, the overall obesity prevalence differed between the studies $(\mathrm{p}<0.001)$ and the agreement was weaker $(\kappa=0.76)$. The abdominal obesity prevalence estimates were significantly different according to the 2 systems both in boys and girls, although agreement reached to 0. 88 which represented an almost perfect agreement level. Thus they concluded that this study provided new BMI and WC percentile curves and reference values for Chinese children and adolescents aged 7-18yrs which can be adopted in future references. Large longitudinal study is still required to show the childhood growth pattern and validate the inconsistencies between different percentile studies [12].

\section{InHawai'i}

Richard., et al. described the recommendations made by the obesty prevention task force in 2013 (CT269) for increasing obe- sity in Hawaii. They described that 57\%of adults are overweight/ obese as are almost 1 in 3 children entering kindergarten. Each year obesity costs Hawaii more than $470 \mathrm{~m}$ \$in medical expenditures alone. Thus these serious human and economic costs underscores the serious need for Hawaii to address obesity now.

Because of urgent need to reverse the current trends in obesity Senate Bill 2778 was signed into law on july6 2012 as Act 269 by Governor Neil Abercombie creating the Childhood Obesity Prevention Task force. The task force was charged with developing policy recommendations and proposed legislation for the 2013 legislature. The task force ultimately identified 11recommendations for the 2013 legislatureand one for the 2014 legislative session. When implementedtogether these recommendations could probably reshape Hawaii school, work, community and health care environments making healthier lifestyles obtainable for all Hawaii residents [13].

\section{In Japan}

Kubo 2014 reviewed the approach to childhood obesity in japan in 2014. They described how POW and BMI were used to evaluate weight in japan. POW is calculated as $100 \mathrm{x}$ (the measured weight -normal weight /normal weight (\%). Normalweight data based on age and sex specific standard body weights for height were obtained from the Ministry ofEducation, Culture, Sports, Science and Technology. POW $>=15 \%$ is considered to be mildly overweight, $>=20 \%$ moderately overweight, and $>=30 \%$ severely overweight in children <6yrs old and $>=20 \%$ mild, $>=30 \%$ moderately and $>=50 \%$ severely overweight in children 6yrs or older. Theoretically POW is not influenced by height, thus it is convenient, highly useful index for comparing time dependent changes. Problem is comparison with other countries is difficult because POW is an index which is only used in japan. Further the japan society for the study of obesity devised criteria for obesity as a morbid state which requires medical treatment in 2002. Diagnostic Criteria for childhood metabolic syndrome was given in 2010 by the Japanese Ministry of health, Labour and Welfare since there were increasing number of children with lifestyle related diseases. Further they gave 3 principles on which treatment of obesity needed to be prioritized and use of 3 principles, other than behavioral therapy had been introduced in their study for the general medical treatment of obesity with out underlying diseases namely diet therapy, exercise and no pharmacotherapy. But preventing obesity is more important than its treatment. Since previous study has shown tracking of overweight status from infancy, it is important to prevent infants 
from becoming overweight. Because of concept of developmental origins of health and disease, preventative measures before birth need to be considered for reduction of incidence of obesity. Though methods needed to approach treatment of childhood obesity change with differences in race or lifestyle therefore those engaged in the medical treatment of childhood obesity worldwide need to contribute to its treatment and prevention by mutual exchange of information [14].

\section{In USA}

Bass., et al. described how concerted efforts had been done in the USA and globally in the last 10years to develop and implement educational, medical and public health interventions designed to attenuate its growth. The success of these efforts was probably responsibe for the plateau and in the prevalence of childhood obesity noted in the last 2 yrs. Though attenuation in overall prevalence of childhood obesity noted is promising, data from the same cohort show a concerning upward trend in the number of children with severe obesity. Consequences of severe obesity can be devastating. As compared to their moderate obesity peers, children with severe obesityare at greater risk for adult obesity, early atherosclerosis, T2DM, metabolic syndrome, fatty liver disease and premature death. The determinants of severe obesity are the same lifestyle, environmental factors, familial and societal risk factors reported for overweight and obesity. Though all these risk factors must be screened for, genetic influences, are distinct considerations, which may have greater bearing especially with early onset obesity. Treatments for severe childhood obesity include lifestyle interventions, specialized low calorie diets and bariatric surgery. Outcomes of these Treatments vary, with bariatric surgery clearly the most successful of the 3 for both short term and longterm weight loss. Severe obesity in children and adolescents remains a challenging health condition. The enormous medical, emotional and financial burden these children and their families endure signals an urgent need to further investigate and standardize treatment modalities and improve outcomes [15].

In Chilea

Further Campos., et al. 2016 tried to determine the accuracy of two international BMI cutoffs for obesity comparing the percentage of fat mass (\%FM) assessed by dual energy XRay Absorptiometry (DXA) in a Chilean sample of children and adolescents. They used two international BMI cutoffs i. e. IOTF and WHO criteria which were those proposed by Interational Obesity task force (IOTF)
[16], which was based on six large international representative samples of different ethnicities (Brazil, China, USA, Great Britain, Hollandand Singapore). This IOTF cutoff is an extrapolation of the adult BMI cutoff points of adult obesity $\left(30 \mathrm{~kg} / \mathrm{m}^{2}\right)$ [17]. The WHO [18] Data set was created using information from the 1997 National Center for Health Statistics-NCHS (from 1-24yrs) in conjunction with information from preschoolers less than 5yrs old from 2006 collected by the WHO The WHO [50] system defines obesity as a BMI>2SD from the mean of the WHO reference population. They studied 280 children and adolescents (125 girls and 155 boys) between 8-17yrs. Weight and Height were measured. BMI was calculated. The\%FM was calculated as assessed by DXA. The receiver operating characteristics (ROC) curve was used to assesss the performance of BMI in detecting obesityon the basis of\%FM. They found a high correlation was observed between\%FM measured by DXA and the Z scores of IOTF and WHO scores on the Chilean adolescents separated by sex ( $r=0.78-0.80)$. Differences occurred in both references i. e IOTF and WHO in relation to the criteria $(\mathrm{p}<0$. 001). Both references demonstrated a good ability to predict sensitivity (between $84 \%$ and $93 \%$ ) and specificity between (83\%and $88 \%$ ) in both sexes of children and adolescents. Thus they concluded that a high correlation was observed between the $\mathrm{Z}$ score of the BMI with the percentage of fat determined by the DXA. Despite this, the classifications using different BMI cutoff points showed discrepancies. This suggested that the cutoff points selected to predict obesity in this sample should be viewed with caution [19].

\section{Role of family environment}

Further Huang., et al. 2017 tried to introduce a framework of the complexities of childhood obesity based on the family environment. They gave a conceptual model which quantifies the relationship and interactions among parental socioeconomic status, family food security level, childs food intake, use of technology by child, childs average amount of sleep, childs school grade, childs physical activity and certain aspects of parental feeding behavior using the structural equation modeling (SEM) concept. Structural models were analysed in terms of direct and indirect connections among latent and measurement variables which lead to the child weight indicator. For illustrating the accuracy, fit, reliability and validityof the introduced framework, real data collected from 630 families from Urumqi (Xin chiang, China) were considered. The framework included 2 categories of data comprising the normal BMI range and obesity data. The comparison analysis between 2 models provi- 
des some evidence that in obesity modeling, obesity data must be extracted from the dataset and analyses must be done separately from the normal BMI range. Thus this study might be helpful for researchers interested in studying childhood obesity modeling based on family environment [20].

\section{Effect on sleep breathing related disorders}

Ma., et al. tried to study sleep related eating disorders (SRBD), which have been identified as a major public health problem closely related to adolescent obesity. They aimed to estimate the prevalence of SRBD and snoring in adolescents in Changchun City, Northeastern China, and tried to evaluate the associated factors in the population. A total of 1955 adolescents aged 11-18 yrs were selected from Changchun City using stratified cluster sampling. Patient and caretakers of children completed the questionnaires, which included demographic characteristics, anthropometric parameters and a pediatric sleep questionnaire (SRBD Scale). Logistic regression was used to analyze the relationship between SRBD, snoring and other factors. They found prevalence of SRBD and snoring in their population were 3. 7\% and 3. 3\%respectively, and the prevalence of overweight and obesity were $12.6 \%$ and $4.9 \%$. Multivariate logistic regression showed that urban residence $(\mathrm{OR}=2.356$, 95\%CI:1. 251-4. 435) and post term birth (OR = 3. 275, 95\%CI:1. 396-397. 683) were significantly associated with SRBD. Preterm birth (OR = 2. 255, 95\%CI:1. 021-4. 980) and parental education level of university and above (OR = 0. 265, 95\%CI:0. 083-0. 850) were significantly associated with snoring. Overweight $(\mathrm{OR}=2$. 063, 95\%CI:1. 062-4. 006) was also related to snoring. Thus they concluded that prevalence of SRBD and snoring were similar to those reported in previous studies. Urban residence and postterm birth were important influencing factors for SRBD; Overweight, highest parental education level (university and above) and preterm birth were key factors affecting snoring in adolescents [21].

\section{Mathematical model for prediction in UK}

WHO identified OW as one of the most serious public health challenges of the 21 st century with 42 million children $<5 y$ rs estimated to be overweight globally Thus a 6 year action plan on childhood obesity having an aim of halting the rise in childhood OW by 2020 was made by the European Union. In 2016, the UK government published a plan of action for tackling childhood obesity, aiming to significantly decrease the rates of childhood obesity within next 10years by supporting healthier choices in children and engaging communities, schools and industry to make food and drink healthier [22].

Evidence exists that the in utero environment induces a response in the fetus, that can cause increased susceptibility to disease later in life [23]. This concept is described as the 'Developmental origins of health and disease (DOHaD). Fetuses which are developing can adapt to unsuitable in utero environment by undergoing structural, physiological and hormonal changes that are of benefit for short term survival, but it affects future health [24], that can be transmitted through generations [25]. The' maternal response hypothesis' reflects that nongenetic evolution has caused a competitive dominance of adipocytes over other cell types in the acquisition along with sequestering of energy in the body, that gets maintained by the simultaneous existence of excess maternal resources and sedentary behavior during pregnancy, that causes continuous fetal metabolism getting dysfunctional [4]. There is transmission in generations, regarding behavioral patterns via socially mediated learning [26], along with postnatal environment affecting the behavior of infants as well as young children based on that of primary caregiver [4]. Hence there is a suggestion that DOHa D include all aspects of environment and all sensitive windows i. e. preconception, pregnancy, early childhood and others yet to be identified [25].

Thus the WHO Commission on Ending Childhood Obesity considered that it is important to address the critical periods in development which include preconception and pregnancy along with treating children that have been found to be obese [27]. Increasing obesity in women of reproductive age affects the health of the mother along with putting the offspring at risk of developing childhood obesity with its consequence additionally [28]. Since there is lack of effective long term treatments, one needs to focus on preventing development of Childhood Obesity [29]. For prevention one needs to identify individuals who are atsincreased risk of obesity extending from childhood to adulthood [31], especially when both parents having obesity [30] exist. A meta-analysis concluded that there is low probability of losing weight without weight loss treatment [32]. Though tracking childhood BMI to adulthood obesity was weaker [20]. Though this tracking childhood BMI extending to adulthood was, in late adulthood there is need for identification of high risk population and acting as early as possible in view of greater adult morbidity along with mortality that is associated with OW in childhood and adolescence. After identification of high risk po- 
pulations, mathematical models on childhood obesity, trajectories which predict energy imbalance that includes excessive energy imbalance including excess energy intake which underlie obesity and thus calculate the magnitude of intervention necessary to get change in weight which can be used to get the intervention. Thus Zauddeen., et al. 2018 aimed to systematically review prediction models for childhood overweight/obesity and critically assess the methodology of their validation and reporting. Studying studies describing prediction model/score for overweight and obesity between 1 to13 years of age they extracted data using the Cochrane CHARMS checklist for prognosis methods. Ten studies were identified which developed (one) developed and validated (seven) or externally validated on existing (two) prediction model. 6/8models were developed using automated variable selection methods. Two studies used multiple imputation to handle missing data. From all studies 30, 475 participants got included. Of the 25 predictors onkly seven were included in $>1$ model with maternal BMI, birth weight and gender the most common. Thus they concluded that various prediction models exist but most have not been externally validated or compared with existing models to improve predictive performance. Mathematical limitations in model development and validation combined with nonstandard reporting restrict the implementation of existing models for the prevention of childhood obesity [33].

\section{Other Causes of childhood obesity}

Genetic factors have been implicated in childhood obesity. The BMI of monozygotic twins correlated well irrespective of whether they get reared together or separately. But correlation of dizygotic twins was shown to be similar to their siblings [34]. Children of obese parents are more likely to become overweight than those of normal weight parents. Kubo., et al. [35] conducted a study on young women in Japan and showed that prevalence of overweight subjects with either one or two overweight parents was high. Additionally children having two overweight parents were slightly more overweight than those with only one overweight parent. Because early humans were exposed to periods of starvation," thrifty genes" responsible for storing fat and decreasing energy consumption have been conserved in the human genome But since this genotype efficiently prepares individuals for periods of famine, it appears to be obsolete in first world societies in which there is constant abundance of food, thus this mismatch between the environment in which the brain evolved and the environment of today has led to widespread chronic obesity. Furthermore Prader Willi Syndrome (PWS), Bardet -Biedel syndrome and Alstrom syndrome are associated with heredity obesity. Because of challenging treatment for parents and caretakers with relentless food seeking behavior like lying, stealing, hoarding food eating inedibles and limited management avenues currently Salehi reviewed how promising additional therapies for hyperphagia are being offered by current research on PWS [36].

Lifestyle choices, like overeating and lack of exercise, have also been implicated in childhood obesity. Formula food and mixed nutrition have been shown to cause greater prevalence of overweight infants than those fed by breast feed [reviewed in ref 2]. Also early start of baby food and excess seasoning has led to an increase in the number of overweight infants. The unwholesome lifestyle and eating habits associated with later childhood and adolescence have also been implicated in the development of obesity like preference for fast food, omitting breakfast, midnight snacking playing video games, attending cram schools, refusing to go to schools, and buying and eating food with pocket money. The link between "screen time" and obesity, cardiovascular risk factors, negative health indicators and physical inactivity has been well documented.

\section{Role of inhibitory central control}

Although it is clear that obesity is driven by multidimensional factors [37], recent studies show that there is a relationship between obesity and neural circuits which are related to inhibitory control function [38].

Inhibitory control implies that capacity to suppress a dominant response which can get triggered by an external cue, or stop any ongoing response or resist distracting stimuli [39]. Importance of inhibitory responses is in suppressing inappropriate or unwanted actions which can interfere with the completion of any motor, cognitive or socioemotional goals. These are must for day to day life like stopping at traffic lights, preventing impulsive behavior or resisting temptation to eat [40]. It is shown that these inhibitory processes get regulated as well as supported by multiple top-down neural connections [41] which possibly might be compromised in people who are overweight/obese.

Research has shown that obese children have deficits in inhibitory control, along with in attention and are impulsive. All these neurocognitive skills belong to executive function, which by definiti- 
on are the abilities which organize and direct cognitive along with emotional responses to provide regulatory control over thought as well as behaviours, recognize errors and engage in planning. There is presence of these executive functions along with their substrates right from infancy but they continue to mature into adulthood. Prefrontal cortex might be responsible for regulating these functions, and one of the important neurotransmitters controlling the same being dopamine [42].

With the availability of big quantities of foods having poor nutritional value, one needs to inhibit eating frequently for maintaining normal weight (NW). Since there may be interindividual variations in inhibitory control, this might modulate the risk for overeating in conditions where high calorie food is present in plenty [43].

There is poor knowledge regarding the underlying mechanisms behind inhibitory control and mechanism of obesity. However it has been observed that obese adult persons who had examination by positron emission tomography showed lower dopamine D2 receptor density in the striatum as compared to NW controls. Decreased availability of D2 receptors was correlated with greater metabolic activity in prefrontal regions, which suggested some mechanism which adds to overeating. This mechanism would be portended by prefrontal pathways involved in inhibitory control $[44,45]$.

Different behavioral measures have been used to study the association between inhibitory control and overweight/obesity (OW) in children. Gaziano., et al. used tasks of frustration and delay gratification in 57 toddlers and observed that measures of inhibition, emotion regulation along with reward sensitivity at 2 years of age, predicted variations in BMI and obesity at 5yrs of age. Based on parent report data with $(n=346)$ in 6-3year old, it was found by Van den Berg., et al. [91] that higher eating scores was predicted by impulsitivity, and that overeating was significantly associated with BMI. Using the Go/No -Go and incompatibility tasks of the Attention Assessment Battery in 177 children between 8-15yr old children, a high impulsivity and higher BMI was found in younger participants as reported by Pauli-Pott., et al [46]. Comparing 12 obese with 12 NW 12 year old children was done by Cserjesi., et al. who observed that performances on measures of sustained attention and perseveration indices as measured by Wiconsin Card Sorting test, were lower in the obese group. Moffitt., et al. studied a cohort of 1000subjects who had been followed right from birth to 32 years and found that self control measures in childhood (rating on observation of children's lack of control and parent teacher, teacher and self reports of impulsive aggression, hyperactivity, lack of persistence, inattention and insensitivity) predicted health problems, including $\mathrm{OW}$, even after controlling for initial socioeconomic status and intelligent quotient [47]. Only 1 study which used electrophysiological measures of cognitive inhibitory control was identified. Electroencephalographic activity (latency and amplitude of P300 wave) using an auditory discriminated task paradigm was used by Tascilar., et al. [48] where 73 children $10 y$ rs old were found to have reduced amplitude in the obese group in contrast to NW group. Also obese children with insulin resistance had lower P300 amplitude as compared to obese children without insulin resistance [48].

Thus Reyes et al hypothesized that a lower ability to detect conflict and a less efficient use of neural resources might underlie poorer performance of OW children on cognitive and motor inhibition tasks. Thus they used behavioral and electrophysiological methods to study the aspects of inhibitory control in OW and NW children. They measured inhibitory control functions in 93 otherwise healthy overweight/obese and 92NW children 10yr using the Stroop Taskand the Go-No-Go task. Event related potentials were recorded during the Go-No- Gotask. OW children showed slower reaction times (1248. 6ms (95\%CI:118. 29-1314. 3) vs 1149ms (95\%CI:1083. $0-1215.1)$ on the Stroop Test, higher reaction time variability $(0$. 25 (95\%CI:0. 22-0. 27) vs 0.21 (95\%CI:0. 19-0. 24) on the Go-NoGotask and decreased P300 amplitude (4. $1 \mu \mathrm{V}$ (95\% (CI:3. 0-5. 2) vs $6.4 \mu \mathrm{V}$ (95\% (CI:5. 2-7. 6) on event related potentials compared with NW children. Thus they concluded that there is altered inhibitory control functions in otherwise healthy overweight/obese children, that might contribute to their excessive food consumption [49].

Many other problems which include an altered appetite center, psychogenic factors and adipose cell hypertrophy have also been linked to the development of obesity. Maternal resource hypothesis and DOHa D has already been discussed in detail.

\section{Management}

Three important principles for general medical therapy i (diet therapy, ii) exercise and iii) no pharmacotherapy. 


\section{Lifestyle interventions}

Lifestyle interventions are recommended as the primary treatment for childhood obesityand generally comprise diet and exercise interventions, which improve the use of behavioral techniques. Succesful lifestyle interventions are based on behavioral techniques as shown by Reinehr., et al. [50], that stresses on development of new eating and exercise habits and promote specific strategies for changing the person's environment. Behavioral therapies include impulse control techniques, self instruction, cognitive restructuring, development of problem solving strategies, behavioral contracts, booster systems, self reflection curves, and model learning via parents. Though lifestyle interventions have a greater likelihood of failing if there is no cooperation by the family and generally not very effective for severely obese children. It should be offered primarily to children with a real chance of success.

\section{Diet therapy}

A child and his caregiver should receive information from a dietician on how to improve their eating habits and types of food they consumed. Then caloric restriction is conducted if needed to confirm the levelof comprehension by child himself and his caregiver. Meal instructions are given based on the records provided by the caregiver, indicating the meals eaten in the past 3 days, including snacks. The child may be hospitalized if necessary. The most important consideration of diet therapy is that the child is growing, hence an increase in bone and muscle mass, and decrease in fat mass, not simply a reduction of body weight is what is needed. Since body height can be extended, an improvement in body weight may be obtained by just maintaining the Same weight. It is important that treatment does not become an obstacle if the patient is taking part in some activities. For e. g if meals are getting served to children at a school canteen, the child can eat the meal. Further cooperation at home is essential and that the caregiver should not force dietary restriction on the child. Cause of childhood obesity has mainly been related to bad lifestyle choices of the adults responsible. An extreme example is it is not reasonable to deny a midnight snack quoting child will gain weight when other siblings are eating the same. Also instructions for children should not be very strict. Even if their are dietary restrictions which are enforced child must receive praise and reward.

\section{Exercise therapy}

A child might not be able to reduce weight by exercise alone. Even more, many overweight children do not enjoy exercise. Stu- dies have shown that forcing overweight child can suddenly result in injuries, which will further cause more weight gain. So it may be beneficial to make an overweight child continue to perform fixed amount of exercise everyday rather than making him take part in group exercise a few days a week. Exercise causing consumption of $100-200 \mathrm{kcal} /$ day is appropriate and aerobics is considered an ideal exercise for fat metabolism. Walking in school or cycling to school is also a good form of exercise. If these can't be performed helping in household chores may be used to increase the level of physical activity which might include cleaning the bathroom, watering the flowerbeds and rearranging tableware after a meal.

\section{Drug therapy}

Generally any form of pharmacotherapy is not advisable to treat childhood obesity. Orlistat, a gastrointestinal lipase inhibitor which decreases fat absorption is the only one approved. Earlier sibutramine was allowed but has been withdrawn because of its CVS adverse effects. Though appetite depressants, like Mazindol may have to be used as an adaptation. It is not recommended unless BMI is $>35 \mathrm{~kg} / \mathrm{m} 2$. Occasionally it may be used for morbid obesity or children with PWS. A Chinese medicine tablet, Bofu tsusho-san decreases body weight by activating brown adipose cells. Some use it for mild obesity. Although use of thylakoids which are more physiological may be of more help [under publication. In a previous study metformin hydrochloride, an insulin sensitizer indicated for insulin resistance in T2DM, might decrease appetite by increasing glucagon like peptide 1 levels, which was found to be effective in reducing weight [reviewed in ref 2].

\section{Bariatric Surgery}

Three main surgical procedures used in treatment of obesity;gastric restriction, a malabsorptive procedure and extended roux en $\mathrm{Y}$ gastric bypass. In principle BS is not to be used but may be needed more and more in morbidly obese children and PWS. [reviewed in ref 3].

\section{Conclusions}

Thus we have reviewed extensively why childhood and adolescent obesity has become a major public health problem of the $21^{\text {st }}$ century. We have tried to cover the criteria being used in different geographic areas which varies from the WHO BMI cutoffs in USA, Hawaii, to BMI and WC percentiles in China, to POW and BMI in japan, to use of International Obesity Task Force (IOTF) and WHO BMI criteria in Chilea, Holland, Singapore. 
Importance has been given to the $\mathrm{DOHaD}$ origin of heath and disease right from in utero state, to infancy feeding and tracking of obesity from in utero into childhood and from childhood to adulthood with obesity from childhood predicting poor prognosis for development of various diseases like T2DM, hypertension, hyperlipidemia and cancer.

Importance of family obesity, environmental factors has also been emphasized besides role of loss of inhibitory control. Role of developing various mathematical models for predicting childhood obesity development for development of strategies for prevention has been discussed.

Management comprises of various lifestyle measure changes, diet therapy, exercise with avoidance of pharmacotherapy as far as possible. Bariatric Surgery as a last resort is important in morbidly obese children and adolescentsto prevent development of various life style diseases which otherwise become difficult to control.

\section{Conflict of Interest}

No conflict of interest for either of authors exists.

\section{Bibliography}

1. Kulvinder Kochar Kaur., et al. "An Update on a Etiopathogenesis and Management of Obesity". Obesity and Control Therapies P 3.1 (2016): 1-17.

2. Kulvinder Kochar Kaur., et al. "Existing and prospective pathways for intervention in treatment of obesity in a novel waya review". MOJ Drug Design Development and Therapy 2.3 (2018) :95-105.

3. Kulvinder Kochar Kaur., et al. "An update on Bariatric Surgery with long term efficacy and its utilization for medical therapy development from the different mechanism of action and other shortcomes to be outcome". BAOJ Surgery 2.4 (2018): 038.

4. Archer E. "The childhood obesityepidemic as a result of nongenetic evolution:the maternal resource hypothesis". Mayo Clinic Proceedings 90.1 (2015) :77-92.

5. Maggi S., et al. "Obesity: Definition and epidemiology. In Multidisciplinary Approach to Obesity; Springer: Heidelberg, Germany (2015): 31-39.

6. Commision on ending Childhood aObesity. "Report of the Commision on ending Childhood; World Health Organization: Geneva, Switzerland (2016).
7. Ellulu M., et al. "Epidemiology of obesityin developing countries. Challenges and prevention Glob". Epidemic of Obesity 2 (2014): 2.

8. Albert KGM., et al. "IDF Epidemiology Task Force Consensus Group. The metabolic syndrome. -A new worldwide definition". Lancet 366 (2005): 1059-1062.

9. Li C., et al. "Recent trends in waist circumference and waist to height ratio among US children and adolescents". Pediatrics 118.5 (2006): e1390-e1398.

10. Fernandez JR., et al. "Waist circumference percentiles in nationally representative samples of African-American, European American, and Mexican American children and adolescents". The Journal of Pediatrics 145.4 (2004): 439-444.

11. Ma J., et al. "BMI percentile curves for Chinese children aged 7to18years in comparison with the WHO and the US Centers for Disease Controland prevention references". Public Health Nutrition 13 (2010): 1990-1996.

12. Song P., et al. "Waist circumference Reference Value s for Chinese School Aged Children and Adolescents". International Journal of Environmental Research and Public Health 13.6 (2016): 589.

13. Richards K., et al. "The Childhood Prevention Task Force (ACT269): Recommendations for Obesity Prevention in Hawai'I". International Journal of Medical Science and Public Health 72.3 (2013) :102-106.

14. Kubo T. "Common approach to childhood obesity in Japan". Journal of Pediatric Endocrinology and Metabolism 27.7-8 (2014): 581-592.

15. Bass R and Eneil I. "Severe childhood obesity:an under-recognized and growing health problem". Postgraduate Medical Journal 91.1081 (2015): 639-645.

16. Cole TJ and Lobstein T. "ExtendedInternational (IOTF) body mass index cut-offs for thinness, overweight and obesity". Pediatric Obesity 7.4 (2012): 284-294.

17. De Onis M. "Development of a WHO Growth reference for school -aged children and adolescents". Bulletin of the World Health Organization 85.9 (2007): 660-667.

18. Must A., et al. "Reference data for obesity:85th and 95th percentiles of body mass index (wt/ht2) and triceps skinfold thickness". The American Journal of Clinical Nutrition 53.4 (1991): 839-846. 
19. Campos RG., et al. "Accuracy of body mass index cutoffs for classifying obesity in Chilean children and adolescents". International Journal of Environmental Research and Public Health 13.5 (2016): 472 .

20. Huang H., et al. "Family environment and childhood obesity:a new framework with structural equation modelling". International Journal of Environmental Research and Public Health 14.2 (2017): 181.

21. Ma Y., et al. "Associations of Overweight, obesity and related factors with sleep related breathing disorders and snoring in Adolescents: A Cross sectional Survey". International Journal of Environmental Research and Public Health 14.2 (2017) :194.

22. HM Government Childhood Obesity: A plan for Action. Department of Health in London:UK (2016).

23. Galjaard S., et al. "Fetal growthand developmental programming". Journal of Perinatal Medicine 41.1 (2013): 101-105.

24. Godfrey KM and Barker DJP. "Fetal nutrition and adult disease". The American Journal of Clinical Nutrition 71 (2000): $13445-13525$.

25. Heindel JJ., et al. "Developmental origins of health and disease:integrating environmentalinfluences". Endocrinology 156.10 (2015): 3416-3421.

26. Jablonka E and Lamb MJ. "Precis of evolution in four dimensions". Behavioral and Brain Sciences 30.4 (2007): 353-365.

27. World Health Organization. "Report of the Commission on Ending Childhood Obesity. Geneva, Switzerland (2016).

28. Hanson M., et al. "Obesity and the health of future generations". The Lancet Diabetes and Endocrinology 4.12 (2016): 966-967.

29. Oude Luttikhuis W., et al. "Interventions foe treating obesity in children". The Cochrane Database of Systematic Reviews 21.1 (2009): CD001872.

30. Power C., et al. "Measurement and longterm healthrisks of child and adolescent fatness". International Journal of Obesity (Lond) 21.7 (1999): 507-526.

31. Durmus B., et al. "Parental anthropometrics, early growth and the riskof overweight in preschool children: the Generation $\mathrm{R}$ study". Pediatric Obesity 8.5 (2013): 1745-1750.
32. Bayer O., et al. "Factors associated with tracking of BMI:a meta regression -analysis on BMI tracking". Obesity (Silver Spring) 19.5 (2011): 1069-1076.

33. Ziauddeen N., et al. "Predicting childhood overweight and obesity using maternal and early life risk factors:a systemstic review". Obesity Reviews 19.3 (2018): 302-312.

34. Stunkard AJ., et al. "The body mass index of twins who have been reared apart". The New England Journal of Medicine 322.21 (1990): 1483-1487.

35. Kubo T., et al. "Predicting obesity in early adulthood in Japanese women". Journal of Paediatrics and Child Health 44 (2007): 33-37.

36. Neel JV. “Diabetes Mellitus: a thrifty genotype' rendered detrimentalby 'progress'?". American Journal of Human Genetics 14.4 (1962): 353-362.

37. Temple JL., et al. "Television watching increased motivated responding for food and energy intake in children". The American Journal of Clinical Nutrition 85.2 (2007): 355-361.

38. Berthoud HR. "Metabolic and hedonic drives in the neural control of appetite: who is the boss?". Current Opinion in Neurobiology 21.6 (2011): 888-896.

39. Neder koorn C., et al. "Why obese children cannot resist food?" the role of impulsity". Eating Behaviors 7.4 (2006): 315-322.

40. Barkley R. "Behavioralinhibition, sustained attention, and executive functions:constructing a unifying theory of ADHD". Psychological Bulletin 121.1 (1997): 65-94.

41. Mostofsky SH and Simmonds DH. "Response inhibition and response selection:twosides of the same coin". Journal of Cognitive Neuroscience 20.5 (2008): 751-756.

42. Luns B. "Developmental changes in cognitive control through adolescence". Advances in Child Development and Behavior 37 (2009): 233-278.

43. Braver TS., et al. "Vive les differences! Individual variation in neuralmechanisms of executive control". Current Opinion in Neurobiology 20.2 (2010): 242-250.

44. Berthoud HR and Morrison C. "The brain appetite and obesity". Annual Review of Psychology 59 (2008): 55-92. 
45. Volkow ND., et al. "Low dopamine striatal D2 receptors are associated with prefrontal metabolism in obese subjects: Possible contributing factors". Neuroimage 42.4 (2008): 15371543.

46. Pauli Pott U., et al. "Association between inhibitory control capacity and body weight in overweight and obese children and adolescents: dependence on age and inhibitory control component". Child Neuropsychology 16.6 (2010): 592-603.

47. Moffitt TE., et al. "A gradient of childhood self control predicts health wealth and public safety". Proceedings of the $\mathrm{Na}$ tional Academy of Sciences of the United States of America 108 (2011): 2693-2698.

48. Tascilar ME., et al. "P300 auditory event-related potentials in children with obesity:is childhood obesityrelated to impairment in cognitive functions?". Pediatric Diabetes 12.7 (2011): 589-595.

49. Reyes S., et al. "Inhibitory control in otherwise heathy overweight 10year old children". International Journal of Obesity (Lond) 39.8 (2013) :1230-1235.

50. Reinehr T. "Lifestyle interventions in childhood obesity". Nature Reviews Endocrinology 9.10 (2013): 607-614.

Volume 2 Issue 9 September 2019

(C) All rights are reserved by Kulvinder Kochar Kaur.,

et al.

Citation: Kulvinder Kochar Kaur., et al. “Childhood Obesity: A Comprehensive Review of Epidemiology, Aetiopathogenesis and Management of This Global Threat of the $21^{\text {st }}$ Century". Acta Scientific Paediatrics 2.9 (2019): 56-66. 